\title{
Virtual Light Touch Contact: a Novel Concept for Mitigation of Body Sway
}

\author{
Keisuke Shima*, Koji Shimatani ${ }^{\dagger}$, Akitoshi Sugie ${ }^{\ddagger}$, Yuichi Kurita ${ }^{\ddagger}$, Ryuji Kohno* and Toshio Tsuji ${ }^{\ddagger}$ \\ ${ }^{*}$ Graduate School of Engineering, Yokohama National University, 79-5 Tokiwadai, Yokohama, 240-8501 Japan \\ Email: shima@ynu.ac.jp \\ ${ }^{\dagger}$ Department of Physical Therapy, Prefectural University of Hiroshima, 1-1 Gakuen-cho, Mihara, 723-0053 Japan \\ ${ }^{\ddagger}$ Institute of Engineering, Hiroshima University, 1-4-1 Kagamiyama, Higashi-Hiroshima, 739-8527 Japan
}

\begin{abstract}
This paper proposes a novel concept called virtual light touch contact (VLTC), which helps to reduce human postural sway in standing and walking states. The VLTC system essentially creates a virtual partition around the user and enables estimation of the virtual force caused when the partition is touched. The resulting force data are fed back to the user's fingertips in the form of vibrotactile stimulation, allowing sensation of the virtual partition in any location. To verify the validity of the proposed method, a prototype system was developed using motion-capture technology and a tactile stimulator, and preliminary experiments involving a healthy subject were conducted. The results indicated that the system is capable of helping to reduce the postural sway of the user during tandem (heel-to-toe) standing.
\end{abstract}

\section{INTRODUCTION}

Parkinson's disease (PD) is a degenerative disorder of the central nervous system in which four motor symptoms (tremor, rigidity, slowness of movement and postural instability) are considered cardinal. The severity of the condition cannot be fully determined from assessment of its symptoms through blood tests or clinical imaging procedures. As the disease increases the incidence of falling among sufferers due to its impairment of motor function, a new system needs to be developed to reduce the risk of falling and support quantitative diagnosis of the disorder. Following on from the techniques already proposed by the authors to support PD diagnosis [1][3], this paper focuses on a support method to reduce the risk of falling during walking and standing.

Mobility aids such as canes and walkers are commonly used to support walking and mitigate balance problems in elderly people, and such physical devices may represent a primary benefit for certain conditions. Jeka [4] also reported that contact cues provided via the fingertips provide information that helps to reduce postural sway in subjects without balance impairments and in patients with bilateral vestibular loss even when the tiny forces applied are physically insufficient to stabilize body movement. These forces are referred to as light touch contact (LTC).

A variety of studies on the effects of LTC have been reported [4]-[8]. By way of example, Riley et al. [5] discussed how a LTC onto the surface of a cloth curtain helped to reduce the postural sway of subjects in a standing state with the eyes closed. Shimatani et al. investigated the effects of LTC by changing the height of a piece of hanging paper touched by subjects [6] and the illuminance level [7] for elderly individuals. Dickstein et al. also showed that LTC helps to reduce postural sway in a walking state [8]. However, users cannot benefit from LTC without a physical partition (such as a cloth curtain) in real environments. The ability to use the effects of LTC in actual environments without a physical partition will support the development of a novel method to reduce postural sway in standing and walking states, thereby helping to reduce the incidence of falling among elderly individuals.

This paper proposes a novel concept called virtual light touch contact (VLTC), which helps to reduce postural sway without the use of physical partitions such as cloth curtains by leveraging motion-capture technology and vibrotactile stimulation. The method involves setting a virtual partition around the user and estimating the virtual force caused when the user touches it. Data on these forces are then fed back to the user's fingertips in the form of vibrotactile stimulation using a compact lightweight stimulator. The user benefits from the effects of LTC without an actual partition, and postural sway is thus reduced with somatosensory input.

This paper is organized as follows: Section II describes the proposed VLTC concept; Sections III outlines the prototype developed for reduction of human postural sway; Section IV presents examples of experiments performed with a healthy subject and examines the validity of the proposed system; and Section V concludes the paper along with a discussion of future study plans.

\section{VirTUAL Light TOUCH CONTACT (VLTC) SYSTEM}

To support the mitigation of body sway in standing and walking states, it must be possible to leverage the effects of LTC in any location. However, such contact requires the user to touch a physical partition and sense forces from it. To overcome this problem, a novel approach based on a VLTC system with the following criteria is proposed:

1. A virtual partition can be set around the user.

2. Contact with the virtual partition can be sensed via the fingertips as with LTC.

3. Body sway and walking motion can be measured in real time.

4. All sensors and actuators are compact, lightweight, wireless and remotely computer-controlled. 


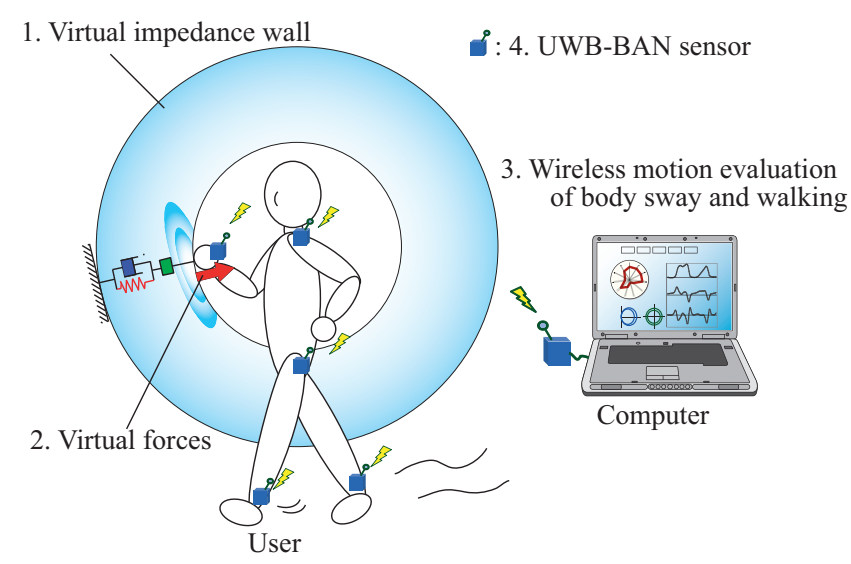

Fig. 1. Concept of the virtual light touch contact system to support daily living

The structure of the proposed concept incorporating the above points is shown in Fig. 1. It involves five stages: (1) determination of fingertip motion properties (3D position, velocity and acceleration); (2) estimation of virtual forces caused by contact with the virtual partition around the user using wireless sensors; (3) conversion of these forces to create vibrotactile stimulation based on human sensory characteristics; (4) stimulation of the user's fingertips to feedback forces; and (5) monitoring and evaluation of body motion and sway characteristics.

A virtual force estimation based on a non-contact impedance model has been proposed in the field of robot manipulation [9]. This method can be used to control a virtual impedance sphere between the object and multiple points set on a robot arm, including the end-effector, so that the virtual interaction between the whole arm and the environment can be considered. In the proposed method, this model is utilized for the creation of a virtual impedance partition between the human trunk and the fingertips to support estimation of virtual forces cased by contact with the partition.

To enable ubiquitous sensation of virtual forces caused by contact with the partition, a tactile feedback system with a compact wearable actuator is required. The authors previously proposed a system for communication between people using a compact lightweight tactile stimulator [10]. In virtual force sensing, a tactile stimulator is attached to the user's fingerpads and stimulation is applied in line with force estimation based on fingerpad sensory characteristics.

For walking support, a compact wireless sensor system and a walking motion analysis system that can be used in real time are required. Ultra-wide band (UWB)-based body area network (BAN) is a wireless communication technology widely applied in medical information communication. In particular, Kohno et al. proposed a positioning and tracking method for sensor nodes involving UWB-BAN [11]. Based on this idea, the proposed method involves the use of UWBBAN devices for monitoring of trunk/fingertip positions and actuation of the user's fingertips. These devices can be easily worn and provide wireless communication to a computer. As

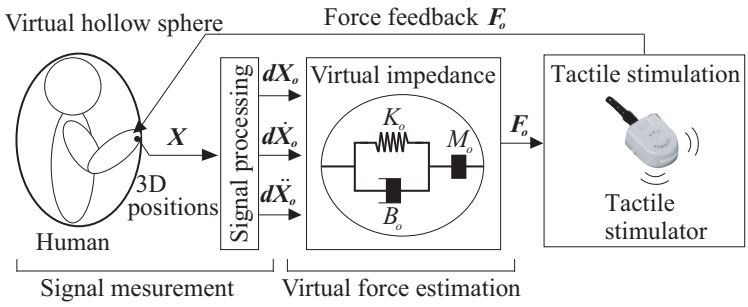

Fig. 2. Prototype system structure

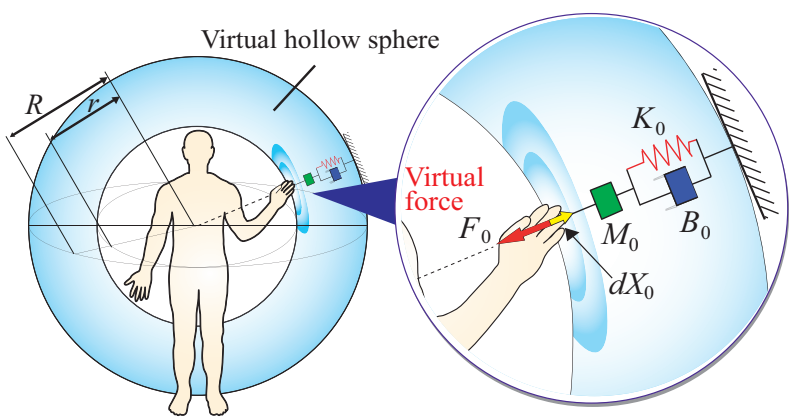

Fig. 3. Schematic representation of the virtual light touch contact concept

the sensors can be worn by the user on a 24-hour basis in any location, body motion and sway data can be collected and transmitted to a computer at a base station using UWB-based BAN technology. The computer extracts motion features and evaluates walking motions in real time.

Based on this concept, the proposed system supports daily living using the VLTC method and enables diagnosis of motor function during walking with BAN technology.

\section{PROTOTYPE}

In this study, the prototype system shown in Fig. 2 was developed based on criteria 1 and 2 listed in Section II above. In this system, 3D motion capture technology using infrared markers is utilized to determine the user's finger and trunk positions, and virtual forces relating to contact with the virtual partition are fed back to the user's fingertips via a vibrotactile stimulator. The details of the system are outlined below.

\section{A. Signal measurement and preprocessing}

Infrared markers are attached to the user's fingertips and trunk, and 3D position vectors $\boldsymbol{X}(t) \in \Re^{3}$ are measured with the origin as the center of the trunk. Fingertip velocity $\dot{\boldsymbol{X}}(t)$ and acceleration $\ddot{\boldsymbol{X}}(t)$ are calculated from fingertip position vectors $\boldsymbol{X}(t)$ using differentiation filters. The Euclidian norm of $\boldsymbol{X}(t)$ is also computed as $|\boldsymbol{X}(t)|$.

\section{B. Estimation of virtual forces}

A virtual partition is set around the user's trunk, and the virtual forces caused when the partition is touched are determined based on a noncontact impedance model [9]. The virtual force estimation method is shown in Fig. 3. With this approach, a virtual hollow sphere with an internal radius of $r$ and an outer radius of $R$ is set with its origin as the center of the trunk. The virtual noncontact impedance between the 
sphere and the center of the user's trunk is considered as shown in Fig. 3, where $M_{o}, B_{o}$, and $K_{o}$ represent virtual inertia, viscosity and stiffness matrices associated with the virtual hollow sphere, respectively. When the user's finger enters the interior of the virtual partition (from the center to the outward direction), the normal vector from the surface of the internal sphere to the finger is given as

$$
\boldsymbol{d} \boldsymbol{X}_{\boldsymbol{o}}(t)=\boldsymbol{X}(t)-r \boldsymbol{n}(t),
$$

where the vector $\boldsymbol{n}(t) \in \Re^{3}$ is defined as

$$
\boldsymbol{n}(t)=\frac{\boldsymbol{X}(t)}{|\boldsymbol{X}(t)|} .
$$

Using the noncontact impedance and the displacement vector $\boldsymbol{d} \boldsymbol{X}_{\boldsymbol{o}}(\mathrm{t})$, the virtual force $\boldsymbol{F}_{\boldsymbol{o}}(t)$ exerted from the virtual partition to the fingertip is defined as

$$
\boldsymbol{F}_{\boldsymbol{o}}(t)=-\left(M_{o} \boldsymbol{d} \boldsymbol{X}_{\boldsymbol{o}}(t)+B_{o} \boldsymbol{d} \dot{\boldsymbol{X}}_{\boldsymbol{o}}+K_{o} \boldsymbol{d} \ddot{\boldsymbol{X}}_{\boldsymbol{o}}\right),
$$

where velocity $\boldsymbol{d} \dot{\boldsymbol{X}}_{\boldsymbol{o}}(t)$ and acceleration $\boldsymbol{d} \ddot{\boldsymbol{X}}_{\boldsymbol{o}}(t)$ are calculated from the displacement vectors $\boldsymbol{d} \boldsymbol{X}_{\boldsymbol{o}}(t)$ using differentiation filters. Via this method, virtual force can be estimated based on contact with the virtual hollow sphere using a noncontact impedance model.

\section{Force feedback using vibrotactile stimulation}

The device utilized to provide virtual force feedback [10], [12] in this system is a tactile stimulator (VBW32C, Audiological Eng. Corp.) that generates tactile sensations against the skin of the user. It has an ideal working frequency of $250 \mathrm{~Hz}$ and a nominal voltage of $2.5 \mathrm{~V}$ RMS. To drive the stimulator, an amplifier unit encompassing a Motorola MC34119 low-power audio amplifier was designed. A tactile stimulator can be driven by sinusoidal or square wave signals; with the former, the vibration level is adjusted using the signal's amplitude; with the latter, it can be adjusted using its duty cycle. In this study, sinusoidal signals were used. A tactile stimulator was attached to the user's fingerpad, and stimulation signals were applied when the virtual partition was touched. Here, the signal amplitude $A_{m}(t)$ was computed according to the norm of the virtual force vector $\boldsymbol{F}_{\boldsymbol{o}}(t)$ as follows:

$$
A_{m}(t)=k\left|\boldsymbol{F}_{\boldsymbol{o}}(t)\right| \text {, }
$$

where $k$ is a constant gain. Via this method, the user can sense the virtual force applied in the air.

\section{EXPERIMENTS}

\section{A. Methods}

To verify the validity of the prototype, experiments with a healthy 24-year-old male subject were conducted. An OpTiTrack (V120: Trio/Duo, Spice Inc.) system was used for 3D motion capture to determine fingertip and trunk positions. A triaxial accelerometer (MA3-04Ac, Microstone Co., Ltd.) was located near the seventh cervical vertebra (C7), and postural sway was computed from the root-mean-square of acceleration. A tactile stimulator was attached to the subject's index fingerpad, and infrared markers were attached to the lateral side of the index fingertip and trunk for position measurement.

The subject was asked to retain a tandem (heel-to-toe) stance with his eyes closed, and was instructed to perform four tasks as follows: (i) VLTC with the right hand (proposed method); (ii) LTC with a piece of paper hanging to the subject's right; (iii) no contact (NC) anywhere and (iv) NC with the hand moving to touch the virtual partition without feedback (NF). The number of trials was 10, and the VLTC parameters were set as $M_{o}=1.0 \mathrm{~kg}, B_{o}=10.0 \mathrm{Ns} / \mathrm{m}$, $K_{o}=50.0 \mathrm{~N} / \mathrm{m}, r=0.4 \mathrm{~m}, R=1.0 \mathrm{~m}, k=0.002 \mathrm{~V} / \mathrm{m}$. The sampling frequency of the $3 \mathrm{D}$ motion capture system and the acceleration sensor was $100 \mathrm{~Hz}$.

\section{B. Results and discussion}

Examples of measured data for task (i) VLTC and (iv) NF are shown in Fig. 4, which plots 3D positions of the right finger, the norm of finger positions, finger motion properties, virtual force vectors $\left|\boldsymbol{F}_{\boldsymbol{o}}(t)\right|$, amplitudes of stimulation signals $A_{m}(t)$ and the RMS of acceleration. The shaded areas indicate time intervals between instances of contact with the virtual partition $(r<|\boldsymbol{X}(t)|)$ by the subject. With VLTC, it can be seen that the virtual force vectors were fed back in the form of vibrotactile stimulation according to the finger motion properties $\left(\left|\boldsymbol{d} \boldsymbol{X}_{\boldsymbol{o}}(t)\right|,\left|\boldsymbol{d} \dot{\boldsymbol{X}}_{\boldsymbol{o}}(t)\right|\right.$, and $\left.\left|\boldsymbol{d} \ddot{\boldsymbol{X}}_{\boldsymbol{o}}(t)\right|\right)$ during these periods of contact. The RMS values for VLTC are clearly smaller than those for NF.

The results for each task are shown in Fig. 5 ((a): average RMS; (b) average standing time), which indicates that the average RMS for VLTC ((i)) was significantly lower than those for NC and NF. Each average RMS value differed significantly at the $1 \%$ or $0.1 \%$ levels as with LTC. The figure also shows that the average standing time for VLTC was longer than those for NC and NF as well as LTC. These results suggest that the effects of LTC can be achieved using the VLTC method for mitigation of body sway. However, note that the center of gravity of the subject during standing and VLTC should be evaluated for more detailed investigation.

\section{Conclusion}

This paper proposed a novel concept for the mitigation of postural sway using a non-contact impedance model and a vibrotactile stimulator. The method can be used to produce the effects of light touch contact (LTC) from contact with a virtual partition based on a non-contact impedance model. Experimental results showed that the approach helps to reduce postural sway during tandem (heel-to-toe) standing without a physical partition as effectively as LTC with such a partition. In future work, the authors plan to investigate the validity of the proposed method with a greater number of subjects including elderly people. A further aim is to develop a novel compact wireless sensor to monitor finger and trunk positions using UWB-BAN technology for walking support. 


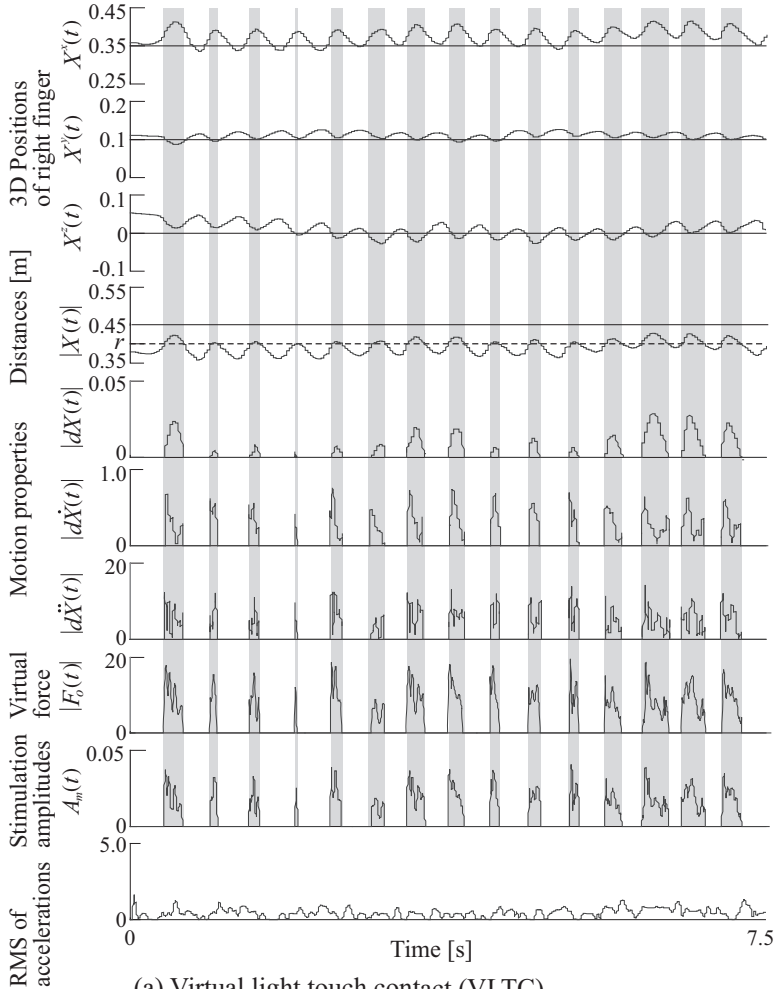

(a) Virtual light touch contact (VLTC)

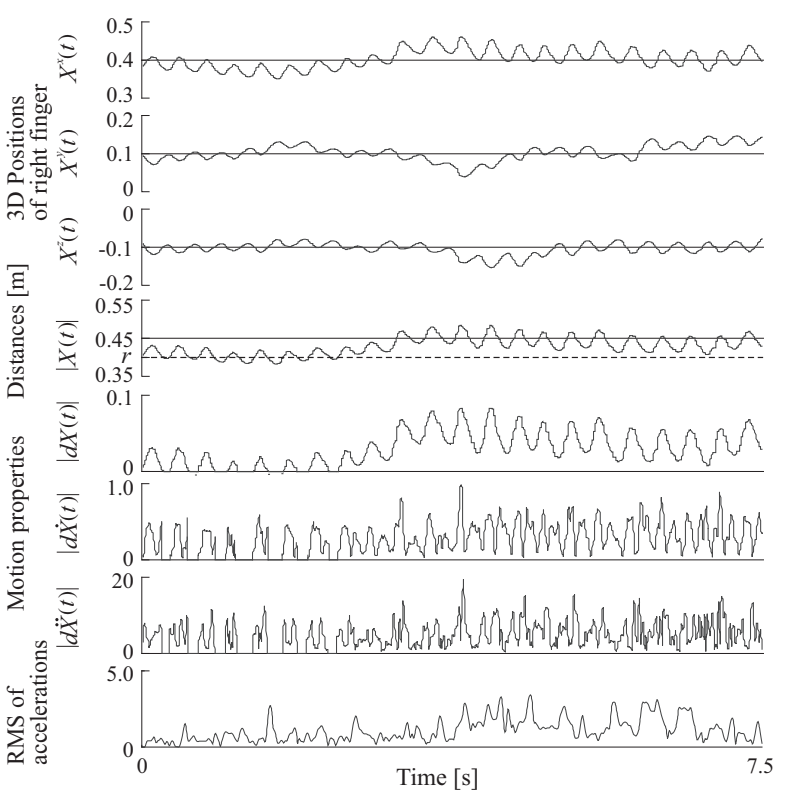

(b) No contact with hand moving to touch the virtual partition without feedback (NF)

Fig. 4. Sample experimental results

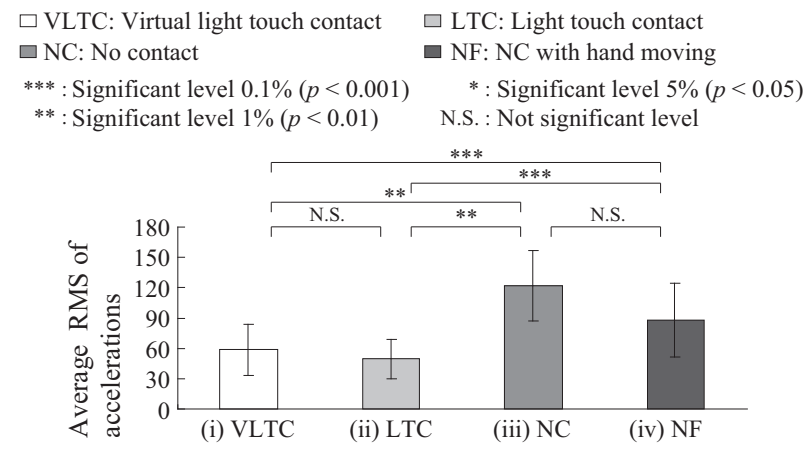

(a) RMS of acclerations

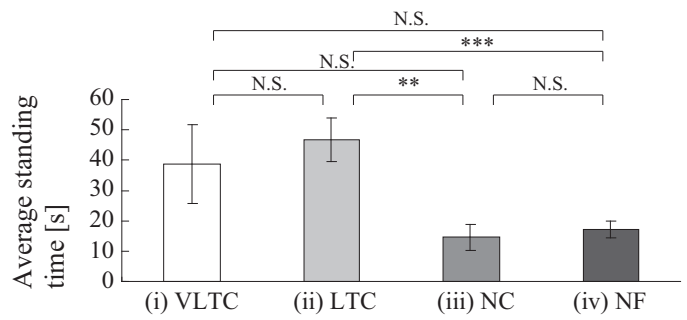

(b) Standing time

Fig. 5. Task result comparison

\section{REFERENCES}

[1] K. Shima, T. Tsuji, A. Kandori, M. Yokoe and S. Sakoda, "Measurement and Evaluation of Finger Tapping Movements Using Log-linearized Gaussian Mixture Networks," Sensors, vol. 9, no. 3, pp. 2187-2201, 2009

[2] K. Shima, Y. Tamura, T. Tsuji, A. Kandori, M. Yokoe, and S. Sakoda,
"Estimation of Human Finger Tapping Forces Based on a FingerpadStiffness Model," Proc. of the 31st Annual Int. Conf. of the IEEE EMBS, pp. 2663-2667, 2009

[3] K. Shima, Y. Tamura, T. Tsuji, A. Kandori and S. Sakoda, "A CPG Synergy Model for Evaluation of Human Finger Tapping Movements," Proc. of the 33rd Annual Int. Conf. of the IEEE EMBS, pp. 4443-4448, 2011.

[4] J.J. Jeka, "Light Touch Contact as a Balance Aid," Physical Therapy, Vol. 77, No. 5, pp. 476-487, 1997.

[5] M.A. Riley, T. A. Stoffregen, M. J. Grocki and M.T. Turvey, "Postural stabilization for the control of touching," Human Movement Science, Vol. 18, No. 6, pp. 795-817, 1999.

[6] K. Shimatani, T. Goto, E. Koge, S. Oki, M. Hasegawa, S. Kanai, T. Ono, and A. Otsuka, "Effects of Differences in Touch Height and Touch Load on Postural Control During Static Standing," Journal of Physical Therapy Science, vol. 23, no. 5, pp. 769-771, 2011.

[7] K. Shimatani, T. Goto, E. Koge, S. Oki, M. Hasegawa, S. Kanai, T. Ono, and A. Otsuka, "Effect of Differences in Room Brightness on Postural Control during Light Touch Contact," Journal of Physical Therapy Science, vol. 24, no. 3, pp. 223-226, 2012.

[8] R. Dickstein and Y. Laufer, "Light touch and center of mass stability treadmill locomotion," Gait \& Posture, Vol. 20, No. 1, pp. 41-47, 2003.

[9] T. Tsuji and M. Kaneko, "Noncontact Impedance Control for Redundant Manipulators," IEEE Transactions on Systems, Man, and CyberneticsPart A: Systems and Humans, vol. 29, no. 2, pp. 184-193, 1999.

[10] E. Akdogan, K. Shima, H. Kataoka, M. Hasegawa, A. Otsuka, and T. Tsuji“The Cybernetic Rehabilitation Aid: Preliminary Results for Wrist and Elbow Motions in Healthy Subjects," IEEE Transactions on Neural Systems and Rehabilitation Engineering, vol. 20, no. 5, pp. 697-707, 2012

[11] Y. Kashiwabara, K. Taniguchi and R. Kohno, "Positioning and Tracking Algorithm Using Node Selection Method in Wireless Sensor Networks," Proc. of IEEE International Symposium on Information Theory and its Applications 2008 (ISITA2008), pp. 944-949, 2008.

[12] J. Lieberman and C. Breazeal, "TIKL: Development of a wearable vibrotactile feedback suit for improved human motor learning," IEEE Transactions on Robotics, vol. 23, no. 5, pp. 919-926, May 2007. 